\title{
A Synthetic study of dispersive GPR data due to multilayer waveguides
}

\author{
Yi $\mathrm{Yu}^{1, *}$; Jianghai $\mathrm{Xia}^{1,2}$;Feng Chen ${ }^{1}$ \\ ${ }^{1}$ Subsurface Imaging and Sensing Laboratory, Institute of Geophysics and Geomatics, \\ China University of Geosciences, \\ Wuhan, Hubei, 430074, China \\ ${ }^{2}$ Hubei Subsurface Multi-scale Imaging Key Laboratory, Institute of Geophysics and Geomatics, \\ China University of Geosciences, \\ Wuhan, Hubei, 430074, China
}

\begin{abstract}
The electromagnetic waves emitted by groundpenetrating radar (GPR) can show apparent dispersion, when they are trapped in thin interlayers whose relative permittivities are large enough. Those thin layers can act as a waveguide. The velocities and thicknesses of the surface waveguide layers and the velocity of substrate layer determine the feature of dispersion. Leaky modes correspond to the complex roots of dispersion equation of the multilayer waveguide. Finite-difference time-domain method is employed to obtain the GPR data sets of two numerical models. Dispersion images of these models are acquired by phase shift method. Moreover, the theoretical dispersion curves of leaky modes and guided modes are calculated. The simulation results illustrate that the dispersion images of multilayer waveguides are more distorted than those of single layer waveguides. It is difficult to recognize separate modes. Consequently, the inversion result would be unreliable. Further researches are required to develop more reliable methods for solving dispersion equation and inversion.
\end{abstract}

Keywords - GPR, dispersion, waveguide,leaky mode

\section{INTRODUCTION}

Variable antenna offset profiles are utilized with groundpenetrating radar (GPR) surveys to investigate the dielectric permittivities of shallow subsurface layers by measuring velocities of electromagnetic wave. If the thickness of a shallow low velocity layer is comparable with the wavelength of electromagnetic wave, GPR data will show significant dispersion and therefore conventional reflection and refractive methods are insufficient to acquire the accurate layer permittivity.

The modal dispersion of electromagnetic wave is highly similar to the dispersion of surface waves in seismic surveys. Analogically, research methods of Rayleigh and Love waves can be used in the research of dispersive GPR data. There are five methods can be employed to get the dispersion image, including phase shift method ${ }^{[11]}$, so phase velocities of waves for different frequencies (dispersion curve) can be obtained. Then, the thickness and the dielectric permittivities of the shallow low velocity layers and the substrate layer can be finally determined by inversing these phase velocities. This is a common and mature research method to process the dispersive GPR and seismic data sets. Extensive researches have been done about the performance of electromagnetic wave in a single layer waveguide ${ }^{[2][5]}$.Moreover, the thicknesses and velocities of the guide layer and the substrate layer of a single layer waveguide can be stably acquired from a joint inversion of dispersive TE and TM GPR data ${ }^{[10]}$.The modal propagation theory of the multilayer waveguides is far more complicated. Recently, plenty researches have been performed for multilayer waveguides, but lots of mysteries like leaky waves still remain. A two layer inversion of dispersive GPR data sets was done by Claudio Strobbia ${ }^{[13]}$ to estimate the soil water content. Moreover, the difficulties and advantages of multilayer waveguide inversion were discussed in that paper. A synthetic study of two-layer waveguides was done by Jenneke Bakker ${ }^{[4]}$, which showed the differences of the dispersion curves between the single layer model and the two-layer model. The inversion result of synthetic and experimental data reveals that the properties of two layer waveguides also can be determined reliably ${ }^{[15]}$. For the waveguides (over two layers), simulation and field observations still need to be done to test the modal propagation theory and the inversion algorism needs to be developed.

The leaky mode is a special phenomenon commonly occurring in multilayer waveguides, and even the waveguide is lossless. In optics, the physical property and behavior of leaky mode are summarized and categorized ${ }^{[12]}$. Moreover, experiments was designed to observe the leaky waves. ${ }^{[6]}$. While the leaky mode is rarely discussed in dispersive GPR data research.

\section{II . Dispersion Equation AND TheticAl Dispersion CURVE}

In the 1960s and 1970s, the optical modes in solid-state waveguides were the subject of intensive study. In optics, The Transfer Matrix Method (TMM) is used as a primary tool for multilayer planar waveguide analysis, which can 
derive the dispersion equation of $\mathrm{TE}$ and $\mathrm{TM}$ modes in a straightforward manner ${ }^{11}$. A lot of computational methods for determining the roots of dispersion equation are designed, such as CIM and ADR ${ }^{[3][7]}$.

The dispersion equation of multilayer waveguide generated by TMM is in this implicit form:

$$
F\left(f, n, \varepsilon_{i}, d_{i}, c\right)=0
$$

$\varepsilon_{i}$ and $d_{i}$ correspond to the relative permittivities and thickness of i-th layer respectively. $f$ is the frequency of electromagnetic wave. $c$ represents the velocity of light in vacuum. The zeros of (1) correspond to the modal refractive index $n$.Then the speed of electromagnetic wave in the waveguide can be readily calculated ( $v=c / n$ ). The modal refractive index could be a complex number, which indicates that the propagation constant, $k=2 \pi f \llbracket n / c$, can also be complex. And therefore the approach for solving this nonlinear equation need allow the accurate determination of all real roots and complex roots.

The strategy of CIM is to structure a polynomial, whose roots are the same with the equation 1 :

$$
p(n)=\prod_{i=1}^{S_{0}}\left(n-n_{i}\right)=\sum_{k=0}^{s_{0}} C_{k} n^{k}
$$

One essential process of this method is to determine the number of zeros $S_{0}$. The argument principle and the residue theorem can be employed:

$$
S_{0}=\frac{1}{2 \pi j} \int_{c} \frac{F^{\prime}(n)}{F(n)} d n=N_{z}-N_{p}
$$

$N_{p}$ is the number of poles and $N_{z}$ is number of zeros inside the region enclosed by the contour $\mathrm{C}$. According to the formula (3), the contour of integral need to enclose all zeros and exclude all poles to obtain $S_{0}$. And the coefficients $C_{k}$ in equation (2) are given via Newton's recursive formula:

$$
\begin{aligned}
& S_{m}=\frac{1}{2 \pi j} \int_{c} n^{m} \frac{F^{\prime}(n)}{F(n)} d n \quad \text { for } m=1,2,3, \ldots S_{0} \\
& C_{k}=\frac{1}{k-S_{0}} \sum_{j=1}^{S_{0}-k} S_{j} C_{k+j}
\end{aligned}
$$

The polynomial (2) can be easily solved by standard techniques, such as Laguerre's method. To solve the equation (3) and (4), the derivative of dispersion equation is needed. Numerical and analytical approaches to acquire $F^{\prime}(n)$ are discussed by Chen et al. ${ }^{[3]}$.

Fig. 1: Summary of CIM to solve dispersion equation

Fig.1 demonstrates the process of CIM to solve the dispersion equation of multilayer waveguide. The real roots of dispersion equation correspond to the propagation constant of guided modes and complex roots are the propagation constant of leaky modes. Energy of a leaky mode will perform the exponential decay when it is propagating along the waveguide boundary, because of the positive imaginary part of its propagation constant. And this is the difference between leaky modes and guided modes ${ }^{[9]}$.To investigate the dispersive of guided modes and leaky modes in the multilayer waveguide, the phase shift method is employed to obtain the dispersion image.

For a single layer waveguide, the dispersion equation can be analytically solved. Moreover, the cutoff frequency and the maximum and minimum speed in phrase-velocity spectra are helpful to distinguish different modes and approximate the permittivities of the guided layer and the substrate layer. But for multilayer waveguides, determining the cutoff frequencies of modes is really challenging. Theoretically, for a lossless multilayer waveguide (the relative permittivity of the substrate layer is smaller than the minimum relative permittivity of guided layers.), the velocities of guided modes in phrase-velocity spectra will also be between $c / \sqrt{\varepsilon_{\max }}$ and ${ }^{c / \sqrt{\varepsilon_{s}}}$ ( ${ }^{\max }$ is the largest relative permittivity of guided layers and is that of the substrate layer).

\section{NUMERICAL RESULTS}

FDTD is utilized for simulating the propagation of TEmode GPR wave in the multilayer waveguide. Two 3-layer models are designed to discuss the dispersive property of guided modes and leaky modes. The grid size is $0.05 \mathrm{~m}$ and the source wavelet is the derivative Gaussian wavelet whose center frequency is $80 \mathrm{MHz}$. The trace spacing of CMP data set is $0.1 \mathrm{~m}$ and the offset is $7.2 \mathrm{~m}$. The parameters of the high-velocity multilayer waveguide are showed in table 1.

\begin{tabular}{|lcc|}
\hline \multicolumn{2}{|c|}{ Table 1.HIGH-VELOCITY INTERLAYER MODELl } \\
\hline Layer number & relative permittivity & thickness(m) \\
\hline Cover layer(air) & 1 & $/$ \\
1 & 20 (wet sands) & 1 \\
2 & 5 (dry sands) & 1 \\
3 & 20 & 1 \\
Substrate layer & 5 & $/$ \\
\hline
\end{tabular}

Dispersion properties of waveguides are principally determined by the permittivities of waveguide. Moreover, to simplify the model, the conductivities of media are set to zero. Similarly, the second model (low-velocity interlayer model) is designed as table 2 shows.

Table 2. LOW-VELOCITY INTERLAYER MODELl

\begin{tabular}{|lcc|}
\hline Layer number & relative permittivity & thickness(m) \\
\hline Cover layer(air) & 1 & $/$ \\
1 & 10 (wet soil) & 1 \\
2 & 20 (wet sand) & 1 \\
3 & 10 & 1 \\
Substrate layer & 5 (dry sands) & $/$ \\
\hline
\end{tabular}



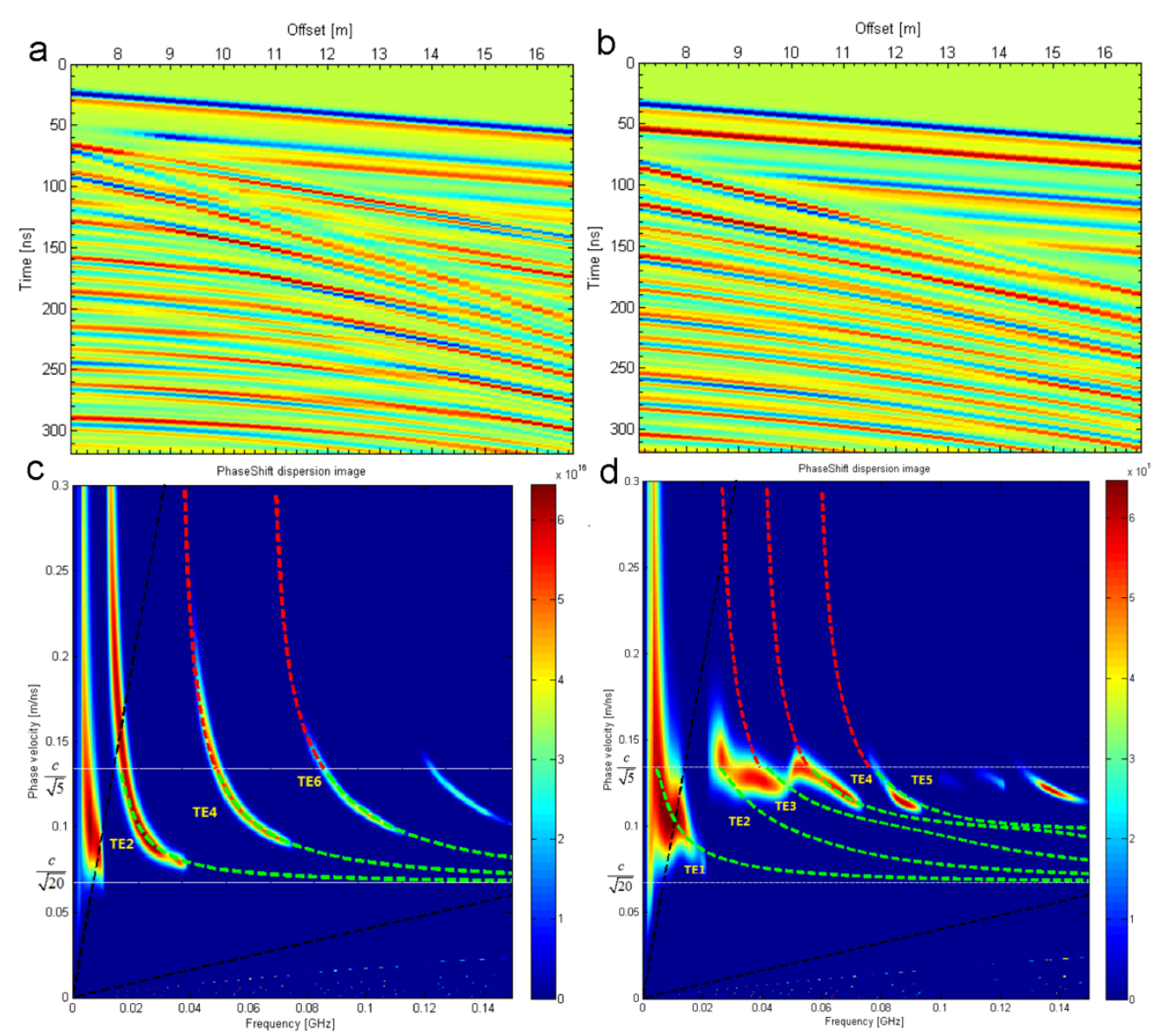

Fig. 2: GPR data sets and diseprsion images of two numerical models ( AGC is applied to data sets to highlight the dispersion. The two black dash lines restrict a reliable zone between the Nyquist wavenumber and minimum wavenumber.Therefore, the data outside of this zone are not discussed in this numerical simulation. The green dash lines denote the theoretical dispersion curves of guided modes and The red dash lines represent the dispersion curves of leaky modes.

Figs. 2a and 2c correspond the data set and the phasevelocity spectrum of the model 1 . For the guided modes, two white dash lines indicate the velocities in the high speed interlayer and the substrate layer, and the speeds of those are in this range. In Fig. 2c, it seems that modes are apparently separate from each other, while the modes are distorted with the neighbor mode in fact. For example, the cutoff frequency of TE5 and TE6 are about $80 \mathrm{MHz}$ and $86 \mathrm{MHz}$ respectively and the modal refractive indexes of them for $150 \mathrm{MHz}$ are 3.679 and 3.661 apiece. Referring to model.2, guided modes in Fig. 2d are extremely tough to identify, because the lowspeed interlayer is not able to trap the energy, which could possibly interfere with other guided waves in other layers and lead to the serious distortion. Furthermore, it is nearly impossible to reconstruct the model 2 from the phasevelocity spectrum, because that cannot provide any velocity information for the low-velocity interlayer.
In Figs. 2c and 2d, three red dash lines are continuous with guided modes. It indicates that velocities of leaky modes are higher than those of guided modes (even can reach to ${ }^{C}$ ) and it is difficult to distinguish leaky modes and guided modes even in the experimental data. Consequently, the inversion result would be unreliable if we mix their dispersion curves. On the other hand, if a joint inversion of guided modes and leaky modes could be performed, the accuracy of inversion will be increased.

\section{CONCLUSION}

In this paper, two numerical models have been designed for GPR dispersive data research and performance of guided modes and leaky modes are analyzed. 
Near-surface soil environment is usually complicated, and therefore we cannot simply interpret shallow soil layers as a single layer waveguide. Moreover, the field dispersive GPR data sets are usually not easy to process, because the modes are distorted with others, just like the two numerical models which are discussed in this paper.

Leaky modes commonly occur in multilayer waveguides. When we are inverting the guided mode, it is essential to get rid of the leaky modes. Also if we could extract some prior information from leaky modes, inversion could be more convinced.

\section{REFERENCES}

[1] Arcone S A. Field observations of electromagnetic pulse propagation in dielectric slabs [J]. Geophysics, 1984, 49(10): 1763-1773.

[2] Arcone S A, Peapples P R, Liu L. Propagation of a groundpenetrating radar (GPR) pulse in a thin-surface waveguide [J]. Geophysics, 2003, 68(6): 1922-1933.

[3] Anemogiannis E, Glytsis E N, Gaylord T K. Efficient solution of eigenvalue equations of optical waveguiding structures [J]. Lightwave Technology, Journal of, 1994, 12(12): 2080-2084.

[4] Bakker J, Van der Kruk J, Bikowski J, et al. Two-layer inversion of dispersive GPR data due to freezing induced waveguides-a synthetic study[C]//Advanced Ground Penetrating Radar (IWAGPR), 2011 6th International Workshop on. IEEE, 2011: 1-5.

[5] Busch S, van der Kruk J, Bikowski J, et al. Quantitative conductivity and permittivity estimation using full-waveform inversion of onground GPR data[J]. Geophysics, 2012, 77(6): H79-H91.
[6] Cassedy E S, Cohn M. On the existence of leaky waves due to a line source above a grounded dielectric slab [J]. Microwave Theory and Techniques, IRE Transactions on, 1961, 9(3): 243-247.

[7] Chen C, Berini P, Feng D, et al. Efficient and accurate numerical analysis of multilayer planar optical waveguides in lossy anisotropic media[J]. Optics Express, 2000, 7(8): 260-272.

[8] Chilwell J, Hodgkinson I. Thin-films field-transfer matrix theory of planar multilayer waveguides and reflection from prism-loaded waveguides [J]. JOSA A, 1984, 1(7): 742-753.

[9] Hu J, Menyuk C R. Understanding leaky modes: slab waveguide revisited [J]. Advances in Optics and Photonics, 2009, 1(1): 58-106.

[10] Kruk J, Streich R, Green A G. Properties of surface waveguides derived from separate and joint inversion of dispersive TE and TM GPR data [J]. Geophysics, 2006, 71(1): K19-K29.

[11] Park C B, Miller R D, Xia J. Imaging dispersion curves of surface waves on multi-channel record[C]//SEG Expanded Abstracts. 1998, 17(1): 1377-1380.

[12] Peng S T, Oliner A A. Guidance and leakage properties of a class of open dielectric waveguides: Part I-Mathematical Formulations [J] Microwave Theory and Techniques, IEEE Transactions on, 1981, 29(9): 843-855.

[13] Strobbia C, Cassiani G. Multilayer ground-penetrating radar guided waves in shallow soil layers for estimating soil water content [J]. Geophysics, 2007, 72(4): J17-J29.

[14] van der Kruk J, Arcone S A, Liu L. Fundamental and higher mode inversion of dispersed GPR waves propagating in an ice layer [J]. Geoscience and Remote Sensing, IEEE Transactions on, 2007, 45(8): 2483-2491.

[15] van der Kruk J, Diamanti N, Giannopoulos A, et al. Inversion of dispersive GPR pulse propagation in waveguides with heterogeneities and rough and dipping interfaces[J]. Journal of applied Geophysics, 2012, 81: 88-96.

[16] van der Kruk J, Jacob R W, Vereecken H. Properties of precipitation-induced multilayer surface waveguides derived from inversion of dispersive TE and TM GPR data[J]. Geophysics, 2010, 75(4): WA263-WA273. 